\title{
Educación social
}

digital: una revisión

sistemática

I+D Educación Social Digital: juventud, ciudadanía activa e inclusión - Septiembre de 2020

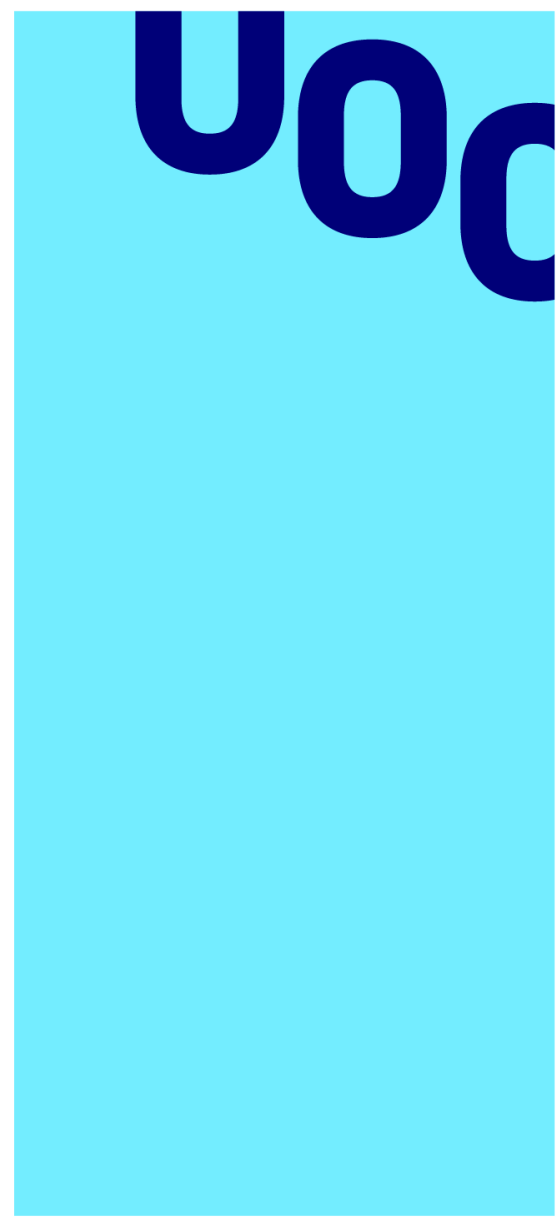

Universitat Oberta de Catalunya

\section{Autores}

Pedro Fernández de Castro

Víctor Sampedro

Daniel Aranda

Segundo Moyano

\section{Índice}

1.- Introducción

2.- Método

3.- Resultados

3.1.- Análisis cuantitativo

3.2.- Síntesis cualitativa

3.2.1.- Ciudadanía y participación

3.2.2.- Alfabetización y competencias

3.2.3.- Educación e inclusión social

4.- Conclusiones

Bibliografía

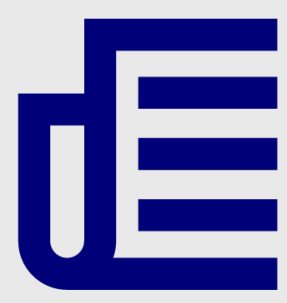




\section{1.- Introducción}

El presente documento se puede considerar el primer paso de una línea de investigación que explora tres aspectos ${ }^{1}$ : cómo los usos de Internet pueden fomentar la ciudadanía activa y la participación política; el desarrollo de una pedagogía crítica en el entorno digital; y el establecimiento de pautas para la educación social digital. La población joven funciona tanto como sujeto de estudio para el análisis de los usos de Internet, como receptor de la pedagogía crítica y educación social digitales.

La pregunta que articula el texto es cómo Internet y el entorno digital influyen en la participación en la esfera pública de las sociedades democráticas, así como motivan el compromiso político, social y cultural de la juventud. Para conocer el estado de la cuestión, realizamos una revisión sistemática de literatura científica de los últimos cinco años en cuestiones relacionadas con la ciudadanía, la alfabetización y la educación social, prestando atención a sus vertientes digitales.

A pesar del incremento de posturas críticas en la última década (Morozov, 2011; Fuchs, 2014; Srnicek, 2016, Lovink, 2019) la literatura dominante tiende a concebir Internet como un espacio ideal para el compromiso político y cívico, en forma de auto expresión y acción, caracterizado por tecnologías digitales "centradas en la gente", "interactivas", "sociales" y "adaptativas", y fundamentales para el desarrollo de una "cultura participativa" (Jenkins, 2016; Frelon, Wels \& Bennet, 2013; Ranieri, Rosa \& Manca, 2016). En particular, considera la información como la primera fase para esta participación, teniendo en cuenta la transformación en la naturaleza de la información (Álvaro \& Rubio, 2016). El consenso gira en torno a que los usos informacionales de Internet tienen efectos positivos en el compromiso democrático (Boulianne, 2009) y que la juventud cuenta con un nuevo canal de comunicación para la participación política. Sin embargo, en los últimos años el aumento en la diseminación de información falsa a través de plataformas digitales, las ya habitualmente denominadas "fake news" (Fox, 2020), han suscitado un debate sobre la necesidad de una intervención para la alfabetización en los entornos digitales con la intención de garantizar los mencionados efectos positivos de la información digital en el compromiso democrático (Mihailidis \& Viotti, 2017; Jones-Jang, Mortensen \& Liu, 2019).

En este contexto, las competencias digitales son un concepto clave, específicamente en las políticas de la Unión Europea. El Marco Europeo de Competencias Digitales para Ciudadanos -DigComp 2.0.- (Vuorikari et al., 2016) y el DigComp 2.1. (Carretero, Vuorikari \& Punie, 2017), señalan cinco áreas competenciales: alfabetización en información y datos; comunicación y colaboración; creación de contenido digital; seguridad; y resolución de problemas. El objetivo es que la ciudadanía pueda acceder y analizar críticamente un constante y diverso flujo de información en el cual fundamentar su participación democrática (Mihailidis \& Thevenin, 2013). En un entorno comunicativo digitalizado marcado por la "desinformación" (información falsa difundida tanto con intención de causar daño como de manera involuntaria) y la "malinformación"

1 Este documento se enmarca en el proyecto de I+D de Generación de Conocimiento de la Agencia Estatal de Investigación "Educación Social Digital: juventud, ciudadanía activa e inclusión” (ESDigital), referencia: PGC2018095123-B-100 
(información basada en hechos empleada deliberadamente para causar daño) la alfabetización mediática y digital o "de datos" (Carmi et al., 2020), es necesaria para una participación plena en una sociedad saturada de medios (Hobbs, 2010).

La educación social, entendida como un "derecho de la ciudadanía que se concreta en el reconocimiento de una profesión de carácter pedagógico, generadora de contextos educativos, y acciones mediadoras y formativas" (ASEDES, 2007), contribuye a promover una sociedad más inclusiva, articulando de manera específica acciones de apoyo al desarrollo personal y empoderamiento de las personas jóvenes. En el trabajo con la juventud, la educación social tiene dos características elementales: trata a los jóvenes como un grupo específico; y actúa, más allá de las aulas, en una multiplicidad de espacios sociales y educativos. El Grupo de Expertos de la Unión Europea del Plan de Trabajo para la Juventud 2016-2018 define la educación social digital como la incorporación "de manera proactiva de las tecnologías y medios digitales en el trabajo con la población joven". La educación social puede jugar un papel relevante en las oportunidades ofrecidas por las tecnologías digitales, considerar los riesgos y las consecuencias, tomar decisiones informadas, y asumir responsabilidades.

El entorno digital representa nuevas oportunidades para la inclusión social. En concreto, los medios sociales se consideran el punto de entrada a Internet para una mayoría "excluida digitalmente" (Correa, 2015). La brecha digital ya no es tanto técnica -la mayoría de jóvenes tienen acceso a Internet y dispositivos tecnológicos- sino de usos y objetivos específicos de los medios y tecnologías digitales. Por eso, las competencias digitales son claves para comprender la desigualdad en la sociedad digital (Morales et al., 2016; Sampedro, 2005). Las aproximaciones críticas en este ámbito exponen el papel de las plataformas comerciales en las dinámicas relacionales en el entorno digital: racionalidad publicitaria, individualismo posesivo y participación promocional (Kaplún, 1998; Kelty, 2008; Gillmor, 2010; Sampedro, 2018).

Este planteamiento debe ser considerado en relación con la noción de "nativos digitales". Este mito conduce a la conclusión errónea de que la población joven ya conoce las tecnologías digitales y no necesita atención en las políticas dirigidas a la desigualdad digital (Sánchez-Navarro \& Aranda, 2012). Al contrario, la creación de contenido y la participación digital requieren combinar habilidades y conocimientos más allá de los usos cotidianos de Internet (Hargittai, 2010; Aranda et al., 2018). El apoyo a la capacidad de actuar responsablemente en el entorno digital, y la respuesta a la necesidad de la juventud de formarse en el entorno digital son esenciales. Por tanto, la educación social debe esforzarse por integrar los medios y dispositivos digitales en sus prácticas y actividades (Middaugh \& Khane, 2013).

\section{2.- Método}

Realizamos una revisión sistemática de literatura académica (Siddaway, Woods \& Hedges, 2018) para ofrecer un análisis cuantitativo y una síntesis cualitativa de la producción en torno a las cuestiones señaladas. Este es un método explícito y replicable 
para identificar, evaluar y sintetizar el conjunto de trabajo relevante producido (Okoli \& Schabram, 2010).

Para iniciar el diseño y planificación de la revisión sistemática se formulan las siguientes preguntas de investigación $(\mathrm{PI})$ :

PI 1.- ¿Cómo influye el entorno digital en la participación -social, cívica y política- de la juventud y la conformación de una ciudadanía activa?

PI 2.- ¿Qué tipo de competencias, habilidades y conocimientos son necesarios para la alfabetización mediática y digital de educadores y jóvenes?

PI 3.- ¿Qué papel puede jugar la educación social digital en el empoderamiento de la juventud, especialmente en la inclusión social de sectores marginados ${ }^{2}$ ?

Estas PI se operacionalizan en palabras clave, utilizadas como términos de búsqueda en las bases de datos para obtener la literatura académica:

digital "youth participation"

"digital citizenship"

digital "media literacy"

digital "youth work"

digital "social education"

digital "youth empowerment"

digital "social inclusion"

digital "(non-formal OR informal) learning"

Tras establecer los términos de búsqueda, elaboramos los criterios de inclusión/exclusión $(\mathrm{Cl} / \mathrm{E})$ :

CI/E 1.- Lapso temporal. Artículos publicados entre 2015-2019.

$\mathrm{Cl} / \mathrm{E}$ 2.- Idioma. Inglés o aquellos cuyos resúmenes, al menos, estén en inglés, aunque el texto esté en español.

Cl/E 3.- Tipo de publicación. Artículos académicos revisados por pares.

2 Nos referimos a marginación por factores socioeconómicos relacionados con lugar de origen, de residencia, género, etnia, nivel educativo, y clase social, entre otros. 
$\mathrm{Cl} / \mathrm{E}$ 4.- Preguntas de investigación. Artículos que, tras la lectura del título y el resumen, respondan a alguna de las tres preguntas de investigación formuladas previamente.

Cl/E 5.- Índice de impacto. Artículos publicados en revistas académicas cuyo ránking esté por encima del 50\% (Q1-Q2) en las categorías de 'Comunicación' y/o ‘Educación', o, en su defecto, en categorías relacionadas con el ámbito de la investigación ('Ciencias Sociales', 'Ciencias de la Información', 'Estudios Culturales', etc).

Cl/E 6.- Categorías de análisis. En base a las preguntas de investigación, establecemos tres apartados de análisis: Ciudadanía Digital, Alfabetización Digital, y Educación Social Digital. Estos apartados están divididos en dos categorías cada uno. Una enfocada en las aproximaciones teóricas a estos conceptos, y otra dedicada a la aplicación práctica en base a los intereses de la investigación.

- Aproximación teórica al concepto 'Ciudadanía Digital'.

- Análisis de la participación social, política, cívica y cultural de la juventud en el entorno digital.

- Aproximación teórica al concepto 'Alfabetización Digital'.

- Análisis de las competencias digitales de educadores y/o jóvenes.

- Aproximación teórica al concepto ‘Educación Social Digital'.

- Análisis de la relación entre empoderamiento e inclusión social de la juventud y uso de tecnologías digitales.

Utilizamos las siguientes bases de datos y cadenas de búsqueda ${ }^{3}$ :

Web of Science: TS=((digital "youth participation") OR (digital "media literacy") OR ("digital citizenship") OR (digital "youth work") OR (digital "social inclusion") OR (digital "youth empowerment") OR (digital "social education") OR (digital "(non-formal OR informal) learning"))

SCOPUS: TITLE-ABS-KEY (( digital "youth participation") OR ( digital "media literacy") OR ("digital citizenship") OR (digital "youth work") OR (digital "social inclusion") OR (digital "youth empowerment") OR (digital "social education") OR (digital "(non-formal OR informal) learning"))

Las búsquedas se realizan el 12 de diciembre de 2019. En ambas bases de datos se aplican los $\mathrm{Cl} / \mathrm{E} 1,2$ y 3 , indicando que incluyan artículos académicos en inglés (título, resumen y palabras clave) publicados entre los años 2015 y 2019 . El conjunto obtenido es de 779 artículos. Tras una revisión manual se identifican y excluyen 39 capítulos de libro. También se eliminan manualmente 41 publicaciones duplicadas, haciendo un total de 699 artículos. Complementamos la búsqueda consultando revistas especializadas en 
Educación Social basada en el conocimiento experto de los autores en este ámbito, obteniendo 7 artículos relevantes. El total de la fase de búsqueda es de 706 artículos académicos. Para la exposición de las fases de búsqueda, filtrado y selección, sirve el modelo de "diagrama de flujo" [Figura 1] desarrollado por el grupo PRISMA (Moher et al., 2009):

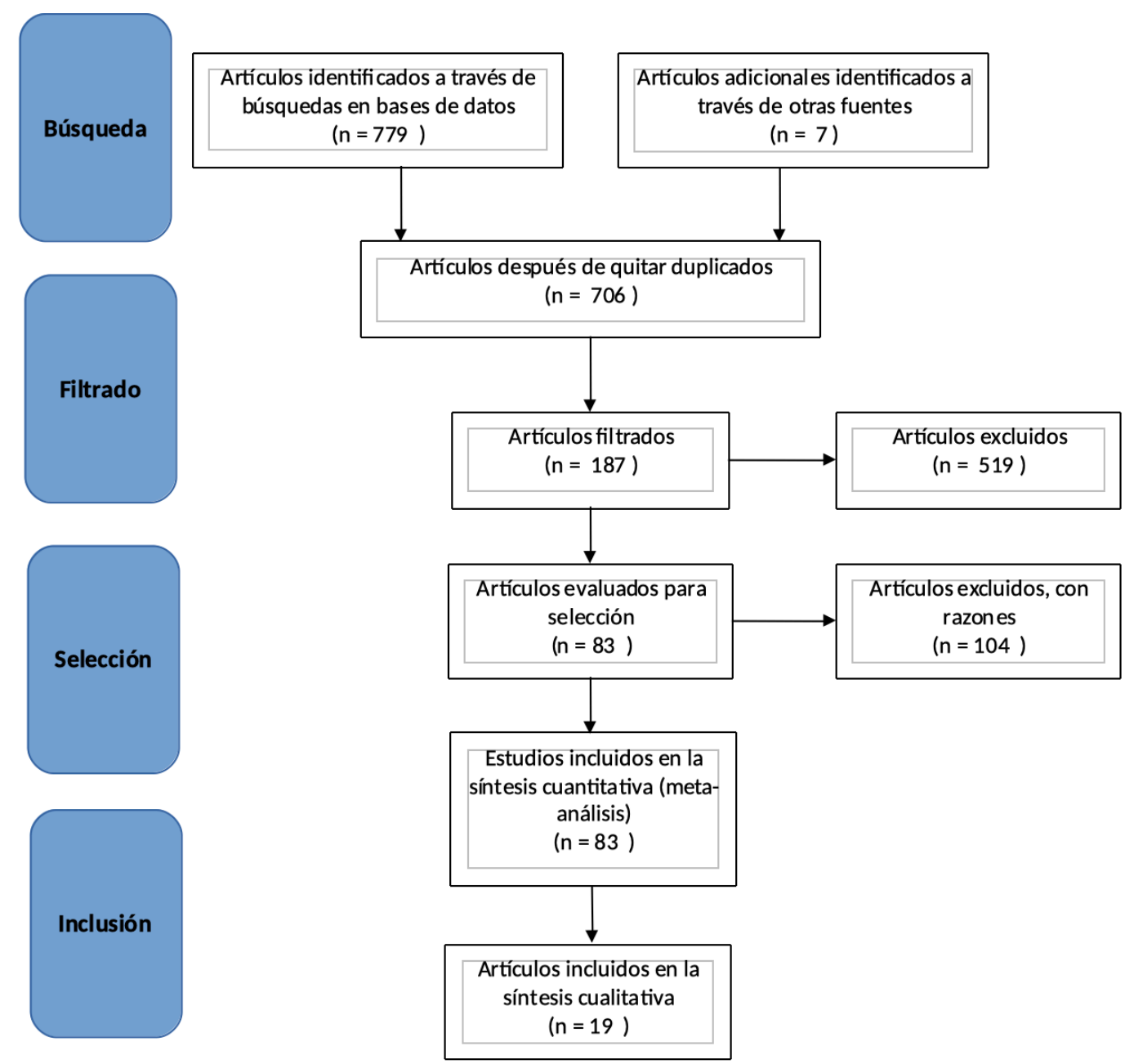

Figura 1. Diagrama de flujo PRISMA (Moher et al., 2009) de la revisión sistemática.

Tras la lectura del título y resumen de los 706 artículos descartamos 519 por no responder a las preguntas de investigación (Cl/E4), manteniendo 187 artículos en el proceso de filtrado y selección. Aplicamos a los 187 restantes el Cl/E5. Para ello, buscamos la revista de la publicación en InCites de Journal Citation Report (JCR) Clarivate- y, si no aparece ahí, en CiteScore -SCOPUS-. Anotamos el percentil asignado en su categoría y el área en el que se enmarca la publicación. Tras este filtro, excluimos 99, manteniendo 88 artículos que pasan a la fase de análisis cuantitativo. Descargamos y hacemos una lectura completa del texto. Cinco de los 88 artículos quedan fuera del análisis cuantitativo, por no haber podido recuperarlos (dos), o porque, se publicaron antes del año 2015 (tres). En total restan 83 artículos.

La siguiente fase tiene dos partes: análisis cuantitativo y síntesis cualitativa. El análisis cuantitativo se realiza a los 83 artículos, mientras que para la síntesis cualitativa se aplica 
el Cl/E6. Tras la lectura de los artículos damos una puntuación en las seis categorías de estudio. Esta puntuación depende de si aborda esa cuestión (1 punto), o, al menos, lo hace parcialmente $(0,5$ puntos). Cada artículo puntúa sobre 6 , y realizamos un corte en los que obtengan, como mínimo, 3 puntos, para garantizar su pertinencia para la investigación: 19 artículos superan este corte para la síntesis cualitativa.

En el análisis cuantitativo de la muestra de 83 artículos se detalla: área de publicación (comunicación, educación, estudios culturales, etc), año de publicación, lugar de publicación y metodología. También atendemos a la puntuación establecida previamente para determinar el peso de cada categoría en la literatura científica del último lustro.

En la síntesis cualitativa, procedemos a la lectura exhaustiva de la muestra de 19 artículos. Realizamos una codificación por colores asociados a las 6 subcategorías. Una vez codificados los artículos, aglutinamos los extractos de cada artículo bajo las subcategorías correspondientes. Así, obtenemos una visión conjunta de los planteamientos existentes en la muestra para cada una. En estos apartados señalamos puntos en común y en conflicto entre los artículos, así como los debates más relevantes en cada uno.

\section{3.- Resultados}

\section{1.- Análisis cuantitativo}

En este apartado tenemos en cuenta los siguientes aspectos de la muestra: área, año y lugar de publicación, metodología, y temas según las categorías establecidas.

Según el área de publicación, entendiendo esta como la categoría -principal- a la que se adscribe el artículo en la indexación de InCites o CiteScore, destacan dos áreas:

Educación (36) y Comunicación (26). Hay que añadir un área más amplia, Ciencias Sociales (13), y otras dos relativas: Pediatría (2) y Ciencias de la Información (4), respectivamente. Por último, hay dos artículos en áreas cercanas: Estudios Culturales (1) y Humanidades (1).

Atendiendo a la distribución temporal del último lustro, observamos un cambio entre los tres primeros años (2015-2016-2017) y los dos últimos (2018-2019). Entre 2015 y 2017 se publicaron, respectivamente, 14, 10 y 14 artículos de los 83 totales. En 2018 aumentó un $50 \%$ el número de publicaciones (21) respecto del año anterior, y 2019 confirma una tendencia al alza con 24 artículos. Esto indica un interés creciente por los temas tratados en esta investigación.

Sobre el lugar de publicación -los países en los que están las universidades desde las que se producen los artículos- advertimos el peso de Estados Unidos respecto del resto. De los 83 artículos analizados, 39 proceden de universidades estadounidenses (47\%). Si se tienen en cuenta los artículos provenientes de Reino Unido (8), Canadá (4) y Australia (3), el predominio de la producción anglosajona es aún mayor, con casi dos tercios (65\%). De Europa continental hay 18 artículos, un 21,7\%, despuntando España (8) con 
un 9,6\%, seguida de Portugal (2) y Eslovaquia (2). Los 11 restantes, un 13,3\%, están repartidos por el globo, destacando Turquía (2) y Corea del Sur (2).

En cuanto a las aproximaciones teóricas y metodológicas, 11 de los 83 artículos (13,3\%) realizan un abordaje teórico (10 discusiones y 1 análisis conceptual). Los enfoques metodológicos mixtos son los más extendidos, utilizados en 21 de los 83 artículos $(25,3 \%)$. Estos incluyen análisis de contenido, encuestas, entrevistas en profundidad, grupos de discusión y etnografías, entre otros. En los artículos que aplican una metodología, destaca la encuesta (20), la investigación "basada en diseño" (9), y el caso de estudio (6).

Por último, en la distribución según las categorías de análisis, observamos una clara desigualdad entre ellas. De los 83 artículos, un 39\% aborda la ciudadanía digital $(15,4 \%)$ y la participación juvenil (23,6\%); un $43,3 \%$ trata la alfabetización digital $(26,2 \%)$ y las competencias digitales (17,1\%); y un $17,7 \%$, la educación social digital $(6,1 \%)$ y la inclusión social y el empoderamiento juvenil (11,6\%). En este punto, es interesante la comparativa con la muestra de 19 artículos de la síntesis cualitativa. Al tratarse de artículos seleccionados en función de la cobertura que realizan de estas seis categorías da resultados más equilibrados entre ellas. Mientras el segundo apartado, alfabetización y competencias digitales, apenas se modifica e incluso aumenta $(44,7 \%)$; hay una disminución del primero, ciudadanía y participación, y aumento del tercero, educación e inclusión social (el primero pasa a representar un $31,7 \%$ y el tercero un $23,6 \%$ ).

Estos resultados cuantitativos muestran desatención a la inclusión social y el empoderamiento en la literatura académica sobre ciudadanía y alfabetización digital enfocadas en la juventud. Además, la escasa producción científica sobre educación social digital confirma la pertinencia de esta investigación, valorizando los resultados cualitativos presentados a continuación.

\section{2.- Síntesis cualitativa}

\subsection{1.- Ciudadanía y participación Ciudadanía digital}

La literatura académica sobre la noción de ciudadanía digital sugiere que se encuentra en proceso de definición. La dificultad para establecer con precisión a qué nos referimos con "ciudadanía digital" muestra que es un término "polifacético" (Panke \& Stephens, 2018) que engloba otros conceptos relacionados (alfabetización, participación, acceso, competencias, normas y valores). Por ello, es abordado desde diversos campos como la educación, la comunicación o las ciencias políticas (Gleason \& von Gillern, 2018). Para que la noción de ciudadanía digital sea útil en el diseño curricular de una disciplina aplicada como la educación social es imprescindible un ejercicio de conceptualización concreto. 
A fin de precisar la noción de ciudadanía digital, una metodología como el análisis conceptual es una herramienta valiosa, especialmente enfocada hacia su uso educativo (Choi, 2016; Gleason \& von Gillern, 2018). Este análisis se cimienta sobre el presupuesto de que la ciudadanía ocupa un rol central en los estudios sociales sobre educación, justificando la necesidad de reinterpretarla en la era digital (Choi, 2016). En la reinterpretación de la ciudadanía en la era digital, conviene mencionar brevemente nociones previas de ciudadanía, distinguiendo entre: "nociones tradicionales", como membresía legal de un Estado-nación con sus respectivos derechos y deberes; y "nociones críticas", en términos identitarios y culturales en el contexto de la globalización. Emerge un debate en torno a la ciudadanía digital como capa adicional de las nociones previas adaptadas a los efectos de las tecnologías comunicativas digitales; o, en cambio, el entorno digital es percibido como un factor determinante que exige una conceptualización propia de la ciudadanía digital.

Choi (2016) concluye de su análisis conceptual una posición intermedia en el debate. Afirma que la ciudadanía digital es un concepto diferente pero no separado de las nociones previas ancladas a la vida offline, reconociendo su relevancia y utilidad en la era digital. Choi (2016) destaca tres elementos comunes a todas las nociones de ciudadanía: "responsabilidad social", "estar bien informado", y "compromiso activo". Una postura semejante expresan Panke \& Stephens (2018) señalando que la esfera digital extiende la ciudadanía "tradicional" y también la transforma. Por contra, Yue et al. (2019) rechazan que la ciudadanía digital sea otra dimensión de la ciudadanía, entendiéndola como una "práctica" en el entorno digital a través de la que se ejerce la ciudadanía.

En cuanto a la definición, Choi (2016) se refiere a la ciudadanía digital como el conjunto de "habilidades, pensamientos y acciones relativos al uso de Internet, los cuales permiten a la gente comprender, navegar, relacionarse y transformarse a uno mismo, a la comunidad, a la sociedad y al mundo". Panke \& Stephens (2018) exponen que no es "algo que tenemos", sino "algo que hacemos", una "práctica reflexiva continuada". En este sentido, coinciden con Yue et al. (2019), quienes asumen la ciudadanía digital como una práctica consistente en la "habilidad para participar online" y una "extensión de la inclusión social". Este último enfoque es, según Gleason \& von Gillern (2018), una de las principales corrientes del término.

En lo que respecta a su encaje con la alfabetización mediática, Yue et al. (2019), en su visión "práctica" de la ciudadanía digital, la incluyen como una subcategoría de la alfabetización mediática. Opuestamente, Choi (2016) desarrolla un marco conceptual con cuatro categorías de ciudadanía digital: "ética"; "alfabetización mediática/informacional"; "participación/compromiso"; y "resistencia crítica". Como señala Choi (2016), este marco ofrece una visión operacionalizable de la ciudadanía digital que sirve para desarrollar instrumentos de medición útiles en el currículum educativo. El propio Choi en sus siguientes trabajos -incluidos en la muestra cuantitativa- desarrolla una escala de ciudadanía digital (2017) y la aplica a educadores (2018).

Nos centramos aquí en la primera dimensión (la ética), ya que las otras tres se corresponden con los siguientes apartados del documento. La categoría de la "ética" de Choi (2016) se divide en tres subcategorías: uso seguro y responsable de las tecnologías comunicativas digitales; conciencia digital; y derechos y obligaciones digitales. Panke \& Stephens (2018) se basan en un informe de Impero Software y el Digital Citizenship Institute (2016) para afirmar que el concepto de ciudadanía digital "refleja nuestra 
necesidad de desarrollar habilidades y perspectivas para una conducta online segura, ética, responsable, inspirada, innovadora e involucrada". Gleason \& von Gillern (2018) identifican este enfoque como otra de las corrientes principales del término.

Por último, Yue et al. (2019) relacionan estos enfoques sobre ciudadanía digital con la juventud. Distinguen dos: enfoque de la libertad, relativo a la habilidad para participar online y la extensión de la inclusión social; enfoque de control, con una perspectiva normativa, entendiendo a la juventud como "no-ciudadana-aún" y, por tanto, necesitada de códigos de comportamiento para convertirse en "buenos ciudadanos". Yue et al. (2019) proponen un tercer enfoque que supere esa dicotomía, centrándose en la "participación cívica". Los ciudadanos digitales, en referencia a Mossberger et al. (2008), son "aquellos que usan la tecnología para informarse políticamente y realizar su deber cívico". Por tanto, la ciudadanía digital no solo se encargaría de las prácticas en el entorno digital, también de cómo estas prácticas se relacionan con sus vidas offline. El paso de la ciudadanía digital al ámbito presencial e institucional a través de sus prácticas es un punto especialmente relevante.

\section{Participación juvenil}

Cabe recordar que la educación social trata a los jóvenes como un grupo con necesidades y aspiraciones específicas. Literat et al. (2018) afirman que los marcos para analizar la participación digital generalmente no se dirigen a la juventud como categoría particular. Literat et al. (2018) no solo entienden a la juventud como una categoría social y cultural. Además de ser definida mediante rangos de edad, puede hacerse también a través de las "fases institucionales de la vida". La juventud consiste en una posición institucionalmente subordinada, marcando la transición a la adultez por la participación en el trabajo y en entornos no-educativos (Literat et al., 2018).

De Lucas \& D'Antonio (2019) señalan tres nociones de juventud relacionadas con los enfoques que la ligan a la ciudadanía digital (Yue et al., 2019), como expone la Figura 2. Primero, la juventud como periodo de espera para la vida adulta. Esto implica que los jóvenes son sujetos pasivos necesitados de guía, justificando un "enfoque de control". Esta primera corriente, según afirman De Lucas \& D'Antonio (2019) es la dominante, y tiene como consecuencia planteamientos jerárquicos, e incluso opresivos y discriminatorios. Segundo, la juventud como agente de ruptura generacional. La juventud entendida como una contracultura que llevaría a vías de conocimiento y creación innovadoras, sustentando el "enfoque de la libertad". Esta perspectiva puede conllevar, como señalan Literat et al. (2018), una asunción acrítica de la participación como inherentemente positiva. Y tercero, la juventud no tendría una condición determinada, dando cabida a ambos enfoques. Consideramos más adecuado el carácter indeterminado de esta tercera propuesta, ya que exige evaluar críticamente la participación juvenil.

Para la relación de la juventud con el entorno digital, Liubiniene \& Thunqvist (2015) sugieren el término "generación digital". Pese a que el concepto de "generación" es valioso para profundizar en la concepción de la juventud, Liubiniene \& Thunqvist (2015) lo abordan superficialmente al fundamentarlo en la noción de nativos digitales. Pawluczuk et al. (2019) proponen un término más práctico al hablar de "juventud digital", entendida como la ciudadanía joven de la era digital, haciendo referencia explícita a la necesidad de 
que los educadores sociales asistan a los jóvenes en su exploración de usos de la tecnología digital.

Un debate relevante es el de las "culturas digitales de la participación" (Cohen \& Kahne, 2015; Ito et al. 2009; Jenkins et al. 2016), marco del que parten Literat et al. (2018) para analizar la participación juvenil digital, entendida como una práctica sociocultural. Jenkins et. al (2016) hablan de "culturas participativas", que cabe entender como aquellas "barreras relativamente bajas para la expresión artística y el compromiso cívico, fuerte apoyo para crear y compartir, y algún tipo de mentoría informal [...] donde los miembros creen que sus contribuciones importan y sienten algún grado de conexión social". Literat et al. (2018) contrapesan el debate señalando cómo los entornos mediados digitalmente pueden permitir o limitar nuevas formas, canales y modos de participación. Destacan la "posición social" (boyd, 2014) y el "capital cultural" (Livingstone \& Sefton-Green 2016) como elementos condicionantes para habilitar o constreñir la participación juvenil digital.

Uno de los conceptos recurrentes en la literatura sobre participación juvenil digital es "compromiso cívico" (Martens \& Hobbs, 2015; Mihailidis, 2018; Panke \& Stephens, 2018; Yue et al., 2019). Martens \& Hobbs (2015) lo entienden en dos sentidos: como la participación política convencional; y como las conexiones de la gente con sus comunidades. Destacan que tanto las experiencias educativas formales como las informales pueden contribuir a construir compromiso cívico, señalando distintas formas de medición como comportamientos, actitudes, percepciones, consumos, y conocimientos. Sobre esta noción y su relación con el entorno digital, ya no en términos de condicionantes sino de efectos, existe un debate alrededor de si fomenta una juventud "comprometida" o "descomprometida". Martens \& Hobbs (2015) mantienen una posición distante y se centran en averiguar qué usos y experiencias contribuyen al compromiso cívico. En cambio, Mihailidis (2018), basándose en trabajos previos, expone que el actual entorno digital copado por las redes sociales conduce a una "brecha de intervención cívica" entre la toma de conciencia y la acción significativa. Para superar este debate entre "utópicos y distópicos", Literat et al. (2018) proponen un marco analítico concreto. Al igual que la investigación de Choi (2016) sobre ciudadanía digital, este marco puede servir para diseñar instrumentos de medición aplicables al currículum de la educación social. El marco de Literat et al. (2018) consiste en cuatro categorías: objetivos, actores, contextos e intensidades.

En cuanto a los objetivos, Literat et al. (2018) distinguen tres dimensiones: individualista/colectivista; expresiva/instrumental; y centrada en el proceso o en el producto. Este punto se asemeja a la categoría de ciudadanía digital "participación/compromiso" (Choi, 2016), con dos subcategorías: macro-formas de participación/compromiso, dedicadas a la intervención en la esfera pública y por lo general más colectivas e instrumentales; y micro-formas de participación/compromiso, por lo general más individuales y expresivas -destacando éstas como las más extendidas. En lo referente a actores, Literat et al. (2018) señalan dos dimensiones: individuos/colectivos; y exclusivos/inclusivos.

Los contextos que diferencia este marco son: formal/informal; y de abajo-a-arriba/de arriba-a-abajo. Aquí, Literat et al. (2018) señalan un punto relevante: la dicotomía a menudo asumida entre lo formal como de arriba-a-abajo y lo informal como de abajo-aarriba no siempre es tal. También apuntan la importancia del rol de los educadores como 
determinantes para los proyectos de participación juvenil, estableciendo los límites de (re)apropiación de la juventud. Por lo que respecta a las intensidades, las dimensiones son: ejecutiva/estructural; y minimalista/maximalista. La primera dimensión es fundamental porque aborda la cuestión del empoderamiento al diferenciar entre participación ejecutiva (ejecutar las órdenes de los adultos que diseñan el plan) y participación estructural (los jóvenes se involucran en el diseño del proyecto). Este eje marca la diferencia entre colaboración y participación (Literat, 2012).

Por último, la idealización de la participación como una "práctica invariablemente empoderante" funciona como cobertura de las carencias en la evaluación del impacto de las iniciativas de participación juvenil digital (Literat et al., 2018). Esta perspectiva margina la comprensión de los retos y oportunidades en términos de empoderamiento e inclusión social.

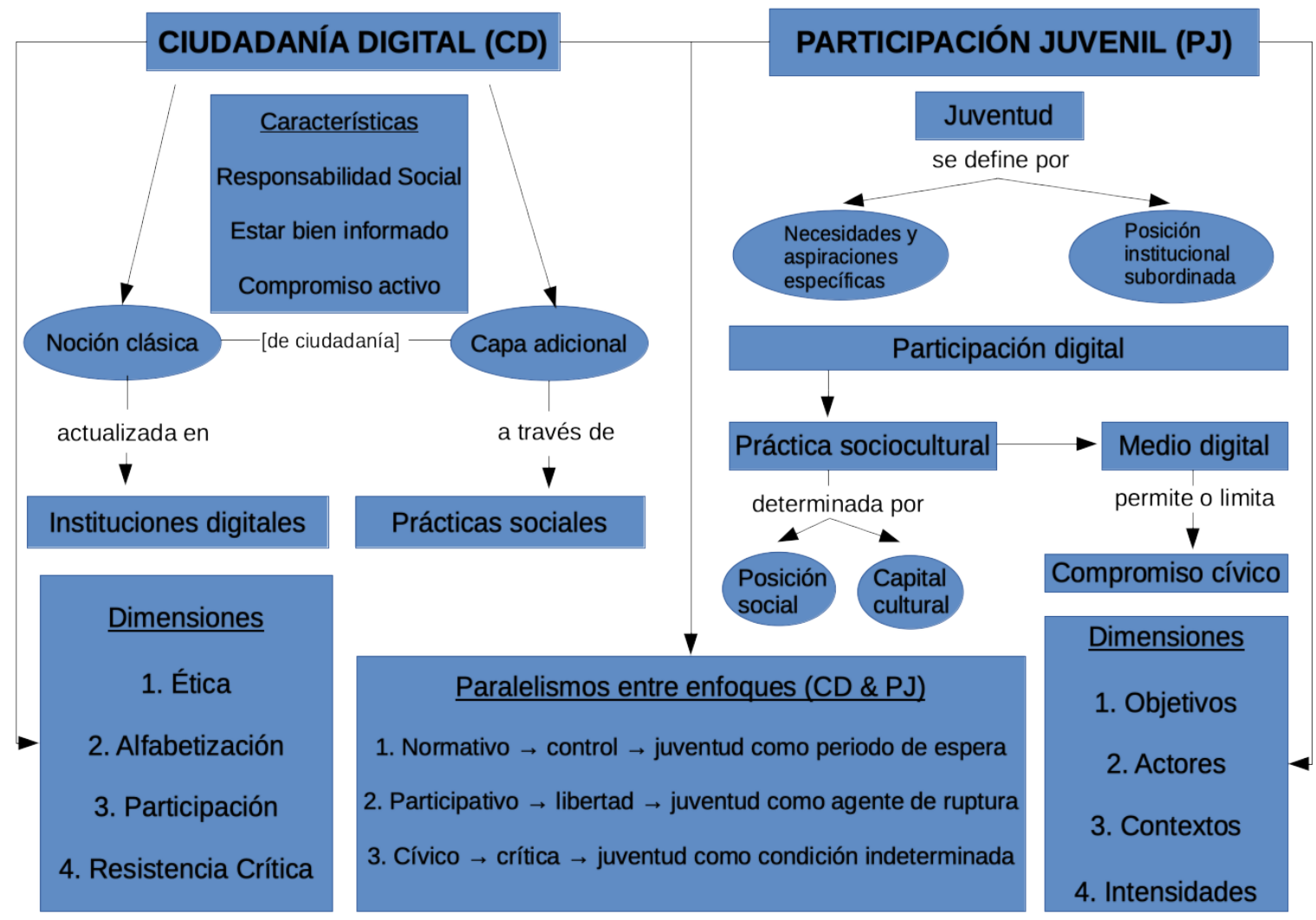

Figura 2. Esquema de relación entre ciudadanía digital y participación juvenil. Elaboración propia.

\subsection{2.- Alfabetización y competencias}

\section{Alfabetización digital}

Perovic (2015) introduce su investigación sobre alfabetización mediática señalando la multiplicidad de términos relacionados con la alfabetización, entre los que destaca "alfabetización mediática", "nuevas alfabetizaciones mediáticas", "alfabetización mediática 2.0", "alfabetización digital”, "alfabetización crítica”, y "alfabetización digital crítica”. Sobre la cuestión del "florecimiento" de los estudios en alfabetización digital, Yue et al. (2019) lo 
explican por el "incrementado consumo cultural de medios digitales y el giro hacia la producción en forma de medios digitales".

El primer concepto que merece atención es, por su condición elemental, "alfabetización mediática". Entre la amplia variedad de artículos sobre alfabetización, hay una definición recurrente que determina a la alfabetización mediática como el "conjunto de habilidades para acceder, usar, crear, analizar y evaluar información en una variedad de formas comunicativas" (Liubiene \& Thunqvist, 2015; Young, 2015; Choi, 2016; Mihailidis, 2018). Esta definición compartida se basa, con ligeras variaciones, en la propuesta de Aufderheide (1993) de hace casi tres décadas.

En este punto es preciso detenerse en el trabajo de Mihailidis (2018). Más allá de la definición básica, se apoya en la estadounidense National Association for Media Literacy Education (ver https://bit.ly/2xBPs|G) para afirmar que la alfabetización mediática "empodera a la gente para ser pensadores y creadores críticos, comunicadores efectivos y ciudadanos activos". Una vez establecido el marco hegemónico, denominado "solucionista", Mihailidis (2018) señala cinco constricciones que restan "relevancia cívica" a la alfabetización mediática: asunción de una "distancia crítica" del ciudadano que no existe; aproximaciones "transaccionales", es decir, priorizar la adquisición de habilidades técnicas que darían un determinado nivel de alfabetización; enfoques centrados en "déficits", como la manipulación informativa; enfoques "centrados en el contenido" y no en las plataformas y cómo su funcionamiento moldea la comunicación; y priorización de la "responsabilidad individual". Además, Mihaildis (2018) considera las normas de la cultura digital actual, que complican la intervención de la alfabetización mediática:

espectacularización cultural; deslegitimación institucional; y brecha de intervención cívica.

En el contexto de la cultura digital actual, es necesario aclarar la noción de "alfabetización digital". Yue et al. (2019) se refieren a ella como las "habilidades y capacidades requeridas por los individuos para participar en una sociedad digital". Complementan la definición referenciando a Gilster (1997), quien acuñó el concepto como la "habilidad de entender y usar información digitalizada", lo que "enfatiza la maestría de ideas más que las habilidades técnicas" (Yue et al., 2019). Panke \& Stephens (2018) definen la alfabetización digital como las "capacidades que encajan en un individuo para vivir, aprender y trabajar en una sociedad digital" (Hibberson, Barrett \& Davies, 2015). Añaden, en consonancia con Gilster (1997), que la alfabetización digital "trasciende las habilidades técnicas aisladas, y abarca una comprensión amplia de los entornos digitales" (Panke \& Stephens, 2018). Pawluczuk et al. (2019) delimita la alfabetización digital como la "habilidad de usar las tecnologías de la información tanto para compartir información como para su creación". En lo que respecta a la juventud, agregan que se trata de un "proceso evolutivo donde los jóvenes acceden, navegan, examinan y producen medios digitales" (Pawluczuk et al., 2019).

Retomando a Mihailidis (2018), sugiere cinco "constructos" para reforzar la intencionalidad cívica de la alfabetización mediática. Frente a la definición básica de alfabetización fundamentada en habilidades (acceder, analizar, evaluar, etc) esta propuesta se centra en los valores que debería promover. En primer lugar, la "intervención", entendida como la posibilidad de empoderar a la gente para actuar en público. En segundo, el "cuidado", la receptividad y la interrelación como ética que se "preocupa por" en lugar de "preocuparse de". Tercero, la "conciencia crítica" como la percepción de la realidad como una situación transformable. Cuarto, "persistencia" para 
aguantar los acelerados flujos comunicativos del entorno digital. Y, en quinto lugar, la "emancipación" como el poder de la participación activa en el diseño de realidades alternativas. Mihailidis (2018) aboga por una "renovación cívica" de la alfabetización mediática, evaluada en función de su "impacto social positivo". El impacto social de intervenciones alfabetizadoras es el objeto de la investigación de Pawluczuk et al. (2019), aunque en el campo de la educación social, como desarrollamos más adelante. Sin embargo, este objetivo compartido entre la alfabetización mediática y la educación social posibilita la interlocución de ambos campos, algo que profundizaremos en próximos trabajos de la presente investigación.

Otra noción relevante es la "alfabetización digital crítica". Pötzsch (2019) desarrolla este concepto desde la premisa de que existe una irreflexión generalizada sobre la implementación de tecnología en espacios educativos. Por tanto, hay una necesidad de fijarse menos en los dispositivos tecnológicos y las habilidades técnicas para hacer un uso eficiente, y más en las capacidades críticas para encajar su uso en las "dinámicas capitalistas, las ramificaciones ambientales, y el empoderamiento individual" (Pötzsch, 2019). Al igual que los "constructos" de Mihailidis (2018), la alfabetización digital no se enfocaría en la formación del usuario en habilidades técnicas para su inserción en el mercado laboral, sino en los valores que fomenten una ciudadanía digital autónoma (Pötzsch, 2019).

Pötzsch (2019) propone tres marcos para desarrollar la alfabetización digital crítica: uso y reflexión sobre alternativas no-comerciales a los productos y servicios corporativos; atender tanto a la historia de las tecnologías digitales como a las prácticas tecnopolíticas; y utilizar expresiones culturales que traten explícitamente cuestiones sobre el poder, la vigilancia y la explotación del entorno digital. Este planteamiento se corresponde con otra de las categorías de ciudadanía digital establecidas por Choi (2016), la "resistencia crítica" y sus dos subcategorías: "crítica de la estructura de poder existente", destacando los valores de la ética hacker; y "activismo político", inspirado en movilizaciones sociales recientes (Primavera Árabe, 15M, Occupies). Pötzsch (2019) argumenta que estos planteamientos inciden en las "tecnologías caja negra" para abrirlas, contextualizarlas, criticarlas y "retener el control democrático sobre sus ramificaciones personales, sociopolíticas, económicas y culturales".

Por último, Tugtekin \& Koc (2019) desarrollan un modelo para evaluar la relación entre la alfabetización mediática, las habilidades comunicativas y la tendencia democrática.

Partiendo de las "nuevas alfabetizaciones mediáticas", establecen dos ejes:

consumo/prosumo; funcional/crítico. Así, emergen cuatro categorías: consumo funcional; consumo crítico; prosumo funcional; y prosumo crítico. Los indicadores que establecen, a modo de competencias, serán abordados a continuación.

\section{Competencias digitales}

Tugtekin \& Koc (2019) aplican diez indicadores para operacionalizar las cuatro categorías que permiten distinguir usos críticos. El "consumo funcional" comprende las habilidades técnicas de acceso; y la capacidad de comprensión de contenidos mediáticos. El "consumo crítico" se compone de tres habilidades: análisis, síntesis y evaluación de mensajes mediáticos. El "prosumo funcional" supone capacidades técnicas para 
producir contenido; habilidad para distribuir mensajes; y competencias para producir diferentes formas de contenido. El "prosumo crítico" incluye la participación como habilidad para interactuar en entornos mediáticos digitales; y la creación, como la generación de contenidos insertos en valores socio-culturales e ideologías teniendo conciencia de los mismos. Young (2015) también utiliza las "nuevas alfabetizaciones mediáticas" y las "culturas participativas" para identificar doce competencias: jugar, actuar, simular, apropiación, multitarea, cognición distribuida, inteligencia colectiva, juicio, navegación transmedia, trabajo en red, negociación y visualización.

Perovic (2015) y Martens \& Hobbs (2015) ofrecen una versión más sintética de la propuesta de Tugtekin \& Koc (2018). Perovic (2015) identifica, aunque no desarrolla, cinco tipos de competencias digitales: acceso, pensamiento crítico, producción creativa, conciencia mediática, y participación cívica. Martens \& Hobbs (2015) definen esos cinco tipos: "acceso", habilidad de tomar decisiones responsables y acceder a la información con capacidad de comprensión; "pensamiento crítico", análisis de mensajes diversos con capacidad de evaluación del contenido; "producción creativa", creación de contenido en diversas formas haciendo uso de herramientas digitales; "conciencia mediática", reflexión sobre la propia conducta basada en la responsabilidad social y la ética; y "participación cívica", realización de acciones sociales, individuales y colaborativas, para compartir conocimiento y resolver problemas. Dentro del mismo marco, Pawluczuk et al. (2019) reducen a tres los tipos de competencias digitales: "uso" (habilidades técnicas); "comprensión" (pensamiento crítico); y "creación", (producción mediática). Tugteking \& Koc referencian a Van Deursen \& Van Dijk (2011) para distinguir entre competencias digitales relativas al "medio" (habilidades instrumentales), y relativas al "contenido" (procesamiento de información y evaluación de contenido).

Estas propuestas desatienden un factor fundamental que señalamos al comienzo: el acceso, no entendido en términos técnicos, sino socioeconómicos (véase la Figura 3). Choi (2016), dentro de la categoría de ciudadanía digital "alfabetización mediática e informacional" divide las competencias digitales en tres subcategorías. El "acceso o brecha digital" contempla a la población diferenciada entre las que disponen de un uso sencillo y seguro, y las que tienen un acceso limitado o nulo, teniendo en cuenta factores como la raza, etnia, edad y nivel educativo. Las "habilidades técnicas", entendidas desde una perspectiva instrumental y consideradas un prerrequisito de competencias avanzadas. Las "capacidades psicológicas" se refieren a las habilidades cognitivointelectuales para procesar datos de manera crítica, las habilidades socio-comunicativas comunicarse e interactuar en entornos digitales, y las habilidades emocionales para manejar sentimientos negativos y desarrollar empatía.

Por último, cabe hacer un añadido sobre la propuesta de Choi (2016). Pötzsch (2019) se apoya en Simanowski (2018) para destacar dos tipos de competencias necesarias para una alfabetización digital crítica: en relación con las habilidades técnicas, la capacidad de "reparar, juguetear, resistir o, simplemente, evitar las tecnologías digitales"; y, en lo relativo a las capacidades psicológicas, la "habilidad para ver la tecnología en contextos locales y globales, y mantener una conciencia crítica para asuntos subyacentes al poder en términos políticos y económicos". 


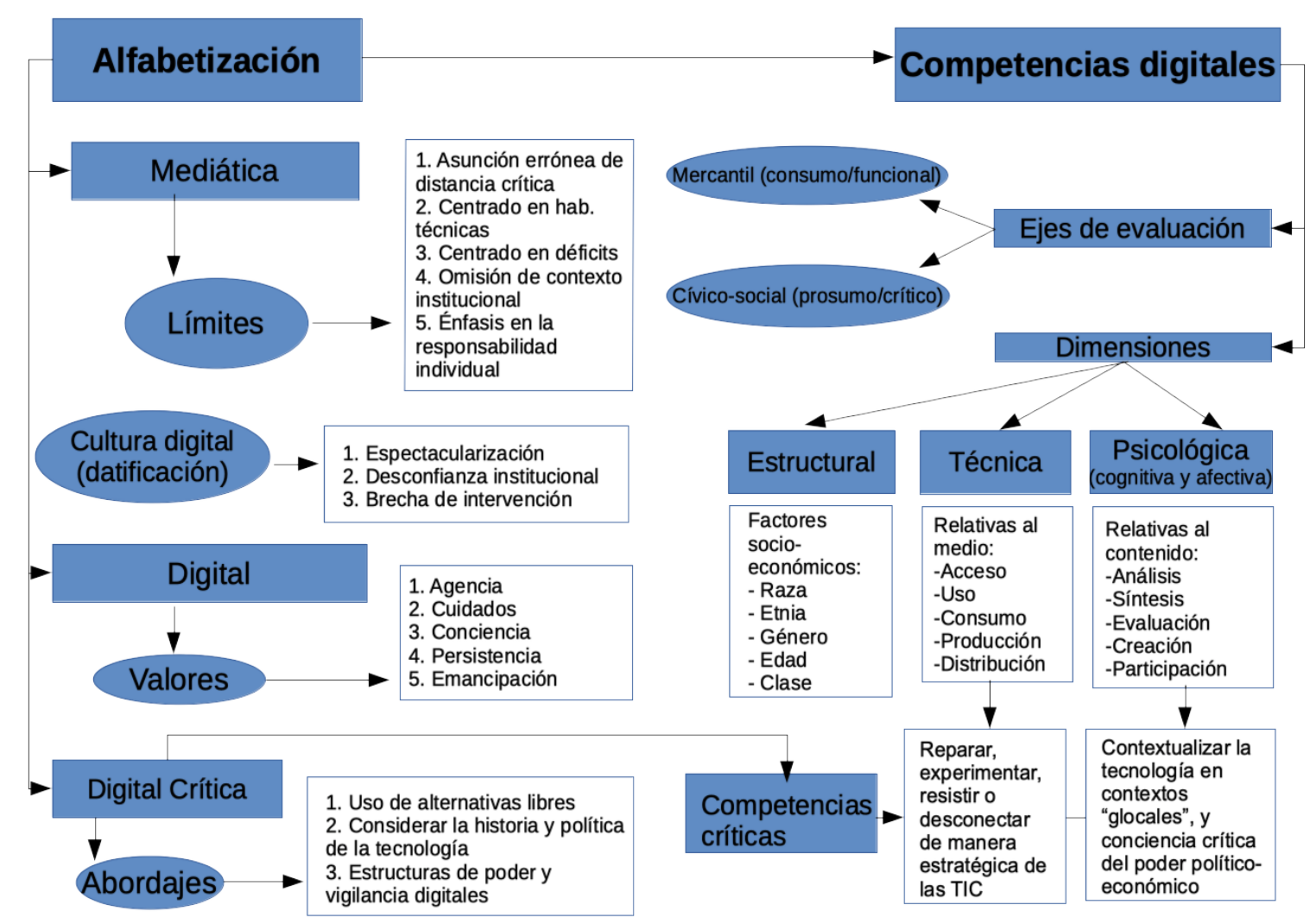

Figura 3. Esquema de relación entre alfabetización y competencias digitales. Elaboración propia.

\subsection{3.- Educación e inclusión social Educación social digital}

Young (2015) afirma que en la última década ha habido un incremento de la literatura científica sobre el uso de las TIC en la educación y práctica de la educación social, al tiempo que destaca otro debate sobre la insuficiente preparación profesional sobre TIC que reciben los estudiantes de educación social. Al contrario, Diaconu et al. (2019), indican una "escasez de literatura" que demostraría "algo de reticencia del campo de la educación social en aceptar las actualizaciones tecnológicas de la profesión". En este sentido, Pawluczuk et al. (2019) exponen que el papel de los educadores con la juventud digital ha sido obviado durante mucho tiempo por la literatura académica, haciendo necesaria más investigación en este campo emergente.

De Lucas \& D’Antonio (2019) exponen que, en la relación entre educación social, TIC, y juventud, una consideración conjunta revela que las "contradicciones que los dividen se incrementan" (ver Figura 4). Esta relación compleja se edifica, por parte de los educadores sociales, en una desconfianza ante la amenaza de "deshumanización" de su trabajo, así como de oprimir la propia actividad profesional; y carencias en competencias digitales. Esta tendencia en cuanto a la implementación de las TIC en la educación social se da en forma de "desvinculación" y "sospecha", lo que De Lucas \& D’Antonio (2019) explican a través del concepto de "solucionismo" (Morozov, 2013). Éste, consiste en la "voluntad de mejorar usando TICs, que se convierten en el medio a través del cual las 
relaciones sociales se articulan, dando soluciones puramente técnicas que parecen resolver los asuntos sociales de manera auto-evidente" (De Lucas \& D'Antonio, 2019).

De Lucas \& D'Antonio (2019) desarrollan un marco crítico para convertir la desvinculación en una oportunidad de que los estudiantes de educación social generen marcos y herramientas para entender y usar críticamente las TIC, mediante la participación activa en su diseño e implementación. Se apoyan en la noción de "coexistencia" de Illich (2011) y destacan la importancia de "alternativas horizontales en el campo de la innovación educativa" (Alonso Puelles et al., 2017). En esta línea desarrollan su trabajo Pawluczuk et al. (2019), con la utilización del término "digital youth work". Afirman que se trata de un término utilizado principalmente en Europa, percibido como parte vital de las prácticas de compromiso juvenil, y referencia a Harvey (2017) para definirlo como el "área del trabajo con la juventud que implementa tecnologías digitales para mejorar los resultados de las iniciativas centradas en los jóvenes". Esta área debe ser entendida como una continuación del trabajo con la juventud y no como un método diferente. Como ejemplos de innovación educativa, destacan alternativas como los clubs de código, clubs de medios participativos, proyectos de campañas y narraciones digitales, o chats de apoyo online.

Diaconu et al. (2019) identifican seis barreras para los educadores sobre tecnología en educación social. Las dos primeras tienen que ver con una falta de apoyo, tanto "institucional" como "técnico". Las dos siguientes se refieren a las condiciones del entorno tecnológico: el "cambio constante" y la "sobrecarga de la opción tecnológica". Las dos últimas son relativas a la formación online, tanto la "escasez de competencias en su diseño", como el "consumo de tiempo en el monitoreo de la interactividad de los estudiantes". Para intervenir en estas barreras, Diaconu et al. (2019) proponen "familiarizar a los estudiantes de educación social con las herramientas esenciales disponibles" de modo que adquieran confianza en el uso de la tecnología para sus prácticas profesionales; y entiendan, se relacionen y se comuniquen mejor con la juventud.

Sin embargo, la propuesta de intervención de Diaconu et al. (2019) redunda en el solucionismo señalado por De Lucas \& D'Antonio (2019). Zorn \& Seelmeyer (2017), partiendo de la necesidad de incluir una asignatura sobre TIC en el currículum de la educación social, afirman que esta educación no debería enfocarse en las aplicaciones actuales "sino en principios más amplios que exploren los impactos sociotécnicos de la innovación tecnológica". Considerando que el currículum de los educadores sociales está saturado, Zorn \& Steelmeyer (2017) abordan la cuestión desde la interdisciplinariedad y el establecimiento de estándares sobre la implementación de las TIC en la educación social. En cuanto al método de aproximación, denominan "aprendizaje basado en investigación" al enfoque pedagógico fundamentado en encontrar "respuestas y soluciones a preguntas más que absorber y repetir conocimientos presentados por el profesor" (Zorn \& Steelmeyer, 2017). De este modo se solventarían las barreras de "cambio constante" y "sobrecarga" del entorno tecnológico, además de contribuir a la mejora en competencias digitales y evaluación en la enseñanza digital.

Sobre los mecanismos de evaluación de las competencias digitales de los educadores sociales, Young (2015) plantea un instrumento de validación basado en las "nuevas alfabetizaciones mediáticas", expuesto anteriormente. Ahí ya señalamos una carencia de este marco: la ausencia del acceso entendido como conjunto de 
desigualdades socioeconómicas. Por tanto, sería necesario desarrollar un instrumento de evaluación que considere la inclusión social basado en las competencias expuestas. Esta herramienta debería considerar el "impacto social" (Pawluczuk et al., 2019), entendido como: "todas las consecuencias sociales y culturales en las poblaciones humanas de cualquier acción privada o pública que alteran las maneras en las que la gente vive, trabaja, juega, se interrelaciona, organiza para cubrir sus necesidades, y generalmente se supera como miembros de la sociedad" (Burdge \& Vanclay, 1995, p. 59). Pawluczuk et al. (2019) destacan dos cuestiones a resolver en esta tarea: el compromiso crítico limitado con el proceso de evaluación de los proyectos para jóvenes y su resultado; y la falta de una definición consistente de dichos procesos. Para ello, proponen que los trabajadores con la juventud digital tengan "un grado de flexibilidad y libertad cuando analicen el impacto social de su trabajo"; y "fomentar la evaluación del impacto social como un proceso crítico, abarcando resultados negativos y positivos y sus desafíos asociados" (Pawluczuk et al., 2019).

Antes de finalizar este apartado, merece mención una cuestión señalada en la introducción como constitutiva de la educación social: los espacios de educación alternativos a las aulas. Pereira \& Moura (2019) se refieren a las escuelas, entendidas como la "instancia más relevante de la educación formal", como "instituciones socioculturales". Éstas se caracterizan por la intervención de múltiples actores, siendo los jóvenes los menos escuchados mientras que los adultos son los "jugadores clave para definir los propósitos de la educación formal" (Pereira \& Moura, 2019). La "falta de atractivo de las escuelas entre los estudiantes", la "pérdida de su posición hegemónica como lugar de aprendizaje", y su "estructura desfasada", son argumentos que señalan que las escuelas, y la educación formal en general, "no se corresponden con las necesidades de la modernidad tardía, y tampoco están sincronizados con las prácticas de la gente joven" (Pereira \& Moura, 2019). Esta visión formal de la educación margina el conocimiento adquirido por los jóvenes en su tiempo de ocio en plataformas digitales con comunicación entre pares. La falta de vasos comunicantes con el exterior del aula lleva a la juventud, en lo que se refiere al entorno digital, a desarrollar estrategias de aprendizaje propias (Pereira \& Moura, 2019).

Panke \& Stephens (2018) entienden el aprendizaje informal como un "recurso sin explotar", desde el rol que tiene en el fomento de competencias enfocadas al compromiso cívico. En su trabajo, Panke \& Stephens (2018) utilizan la herramienta pedagógica del edublogging, destacando sus oportunidades para el aprendizaje informal, así como las "constantes oportunidades a las que ha contribuido en la academia". En lo relativo a la academia, De Lucas \& D'Antonio (2019) señalan que la universidad, donde convergen la educación social, las TIC y la juventud, puede ser un espacio de experimentación para una coexistencia donde la potencialidad y creatividad de la juventud participen en el diseño de programas relacionados con las TIC, y la juventud interactúe con la academia de manera más horizontal y activa.

\section{Inclusión social}

En lo relativo a la inclusión social de la juventud la noción clave es el "acceso/brecha digital" (Liubiniene \& Thunqvist, 2015; Choi, 2016; Garmendia \& Carrera, 2019). Garmendia \& Carrera (2019) parten del creciente foco en la inclusión digital de grupos 
vulnerables, según la premisa de que la "exclusión social puede contribuir a la exclusión digital" (Salemnik, 2016). La discusión sobre la brecha digital se amplía para considerar no solo su aspecto técnico, sino el rol fundamental de los factores socioeconómicos en el uso limitado o nulo de aplicaciones digitales. Al igual que Choi (2016), Garmendia \& Carrera (2019) atienden a la raza, el género, la etnia y la clase social como elementos de influencia. Liubiniene \& Thunqvist (2015) proporcionan un añadido al relacionar la brecha digital con la alfabetización mediática, concretamente en lo que respecta a la "generación digital".

Liubiniene \& Thunqvist (2015) abordan el impacto de los factores socioculturales y socioeconómicos desde el concepto de la "sociedad red" (Castells, 2010). El rápido crecimiento de la sociedad red modifica aspectos cotidianos de la vida, con lo que el "entendimiento tradicional de la estratificación social cambia con el avance de una élite emergente" (Liubiniene \& Thunqvist, 2015), acuñada como "redocracia" (Bard \& Jan Söderqvist, 2002). Ésta se define como la "clase alta global que basa su poder en la ventaja tecnológica y las habilidades relacionadas con la red", de la que podría depender el "futuro de todas las estructuras sociales y sus funciones, desde la política a la economía, desde los modelos de consumo a la cultura, incluso a la construcción de una identidad social" (Liubiniene \& Thunqvist, 2015).

Liubiniene \& Thunqvist (2015) indican que no basta con desarrollar la infraestructura tecnológica, es prioritario "asegurar el desarrollo económico, educativo y sociocultural de todos los segmentos de la población". Garmendia \& Carrera (2019) se apoyan en Kleine (2013) para aseverar que en las "tecnologías para el desarrollo", el "desarrollo es el objetivo y las tecnologías los medios para lograrlo", además de que debería ser visto más como un proceso que como un resultado. Siguiendo con Kleine (2013), esta perspectiva necesita un "proceso abierto de deliberación donde poner las visiones de la gente cuyas vidas son afectadas en el corazón del proceso de desarrollo". Garmendia \& Carrera (2019) añaden que para que este desarrollo contribuya al bienestar de la infancia y la juventud, el abordaje del acceso digital debe entenderse como un derecho.

Por último, Garmendia \& Carrera (2019) reclaman una mayor inversión y voluntad del sector público con la alfabetización digital, para "minimizar la desigualdad y la brecha de conocimiento y promover una mayor justicia social" (Stoilova, Livingstone, \& KardefeltWinther, 2016). Además, para esta tarea la financiación debería "fomentar actividades educativas fuera del sistema escolar formal" (Garmendia \& Carrera, 2019). Las iniciativas no-formales contribuirían al desarrollo de competencias digitales enfocadas a la inclusión social de estratos periféricos y marginados de la juventud en entornos en los que aprender conjuntamente. 


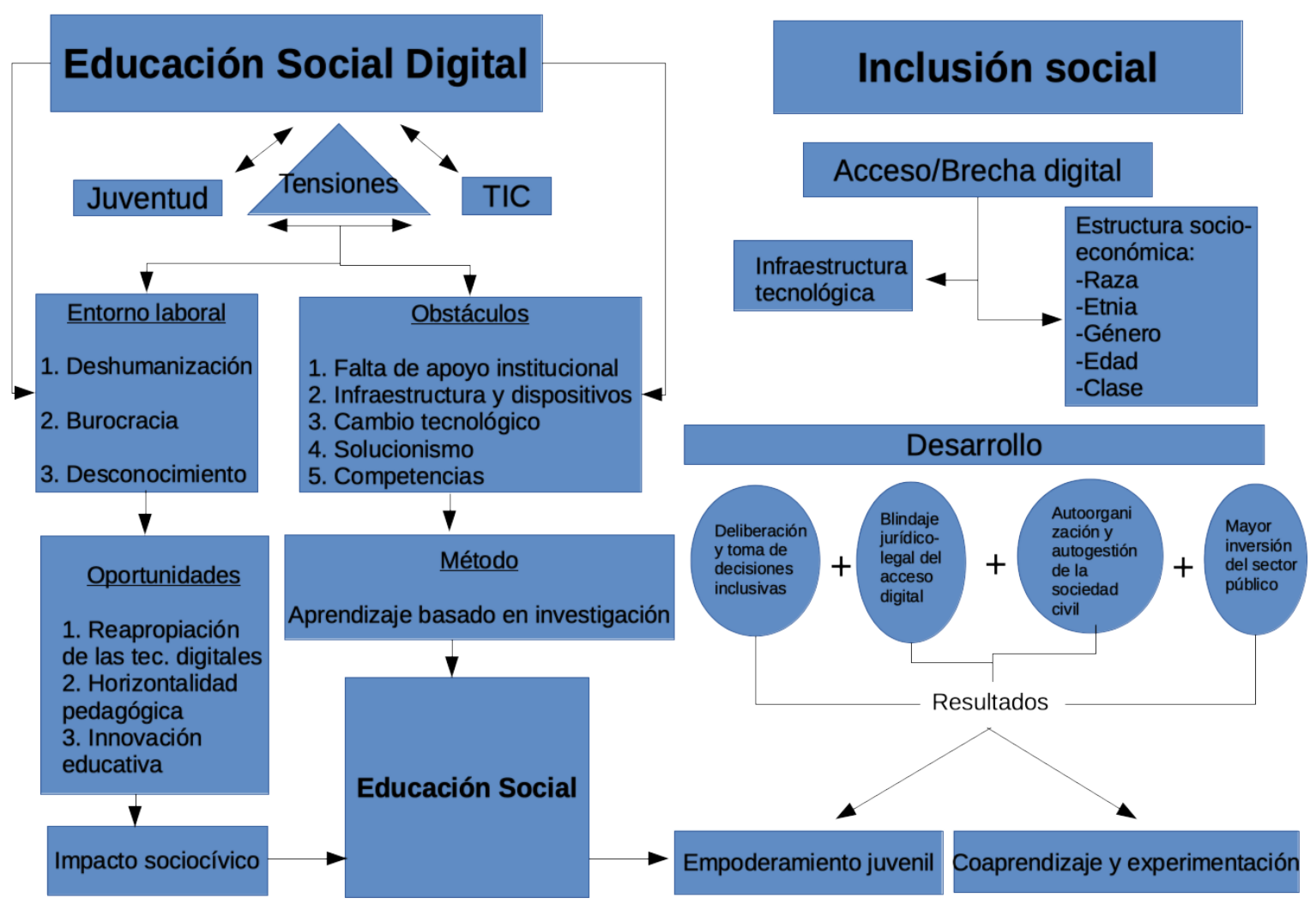

Figura 4. Esquema de relación entre educación social digital e inclusión y empoderamiento juvenil. Elaboración propia.

\section{4.- Conclusiones}

Esta revisión de literatura científica pretende ofrecer una panorámica de los debates académicos más recientes -en el último lustro- en torno al compromiso cívico de la juventud y su participación política, social, cultural y económica en la esfera pública a través de Internet y las tecnologías digitales. Esta exposición parte de que la alfabetización digital y sus competencias asociadas son una vía de acción propicia para fomentar la ciudadanía activa en la juventud. Y también de que la educación social es el ámbito de intervención en el que trabajar con la juventud en general, y, en particular, con sectores marginados por factores socioculturales y socioeconómicos (raza, etnia, lugar de origen, género, clase, etc). De este modo, una educación social digital puede contribuir a la inclusión y el empoderamiento de la juventud lo que, a su vez, redundaría en un fortalecimiento del compromiso cívico y los principios democráticos de la sociedad.

Para ello, diseccionamos la cuestión en tres apartados de análisis: (1) la noción de ciudadanía, con su necesaria actualización y reinterpretación en la era digital, y, más precisamente, la participación digital de la población joven; (2) la alfabetización en medios digitales, concretada en un conjunto de competencias tanto técnicas como intelectuales; y (3) la propia educación social digital, especialmente en lo relativo a la evaluación de su impacto en términos de inclusión social y empoderamiento juvenil. 
Ciudadanía y juventud se toman como conceptos de referencia al constituir tanto el objetivo (la conformación de una ciudadanía activa) como el sujeto (la juventud) de la investigación que a la vista de esta revisión bibiliográfica se revela como la más pertinente de realizar $y$, al mismo tiempo, la más desatendida o pendiente de ser abordada. En cuanto a la noción de ciudadanía, Internet y las tecnologías digitales suponen un conjunto de transformaciones estructurales suficientes como para requerir una reinterpretación en el marco de la era digital. Sin embargo, es preciso mantener el concepto de ciudadanía digital ligado a las nociones offline, para determinar las cuestiones en las que estas continúan siendo válidas, y cuáles necesitan actualización. Así, es posible conceptualizar la ciudadanía digital tanto en su vertiente estrictamente online, como en su interrelación con el entorno analógico. Por otra parte, la juventud, como una categoría sociocultural con necesidades, aspiraciones y miedos específicos, puede entenderse, como es habitual, en términos etarios, pero también como una posición institucional subordinada. Esta situación condiciona la participación digital de la juventud, entendida como una práctica sociocultural en los medios digitales y que viene o se ve determinada por la posición social y el capital cultural, y su compromiso cívico en tanto que ciudadanos activos.

En lo que respecta a la relación entre la ciudadanía digital y la participación juvenil, emergen tres corrientes principales, no necesariamente excluyentes entre sí, pero que marcan la intervención social en proyectos con población joven. (1) La primera tendencia, predominante en los espacios educativos formales, es la "normativa". Parte de una concepción de la juventud como un periodo de espera para la vida adulta y los jóvenes como sujetos pasivos y necesitados de guía. Por tanto, justifica un "enfoque de control" que exige la impartición de códigos de conducta éticos desde una perspectiva vertical. (2) La segunda corriente, la "participativa", comprende a la juventud como un agente de ruptura e innovación, por lo que ha de posibilitarse su participación plena con la mínima intervención adulta necesaria. Este "enfoque de la libertad" corre el riesgo de asumir la participación como inherentemente positiva, ocultando posibles efectos perjudiciales y reduciendo la capacidad de evaluación de las prácticas juveniles. (3) Esta cuestión se atajaría en la tercera corriente, la "cívica", caracterizada por un "enfoque crítico" que hace hincapié en el caracter indeterminado de la juventud (ni sujeto pasivo ni agente activo per se), y la necesidad de un permanente y exhaustivo análisis de la participación digital juvenil en términos de su impacto social y cívico.

Cada una de estas tendencias implica un conjunto de relaciones de la juventud con la educación (social) y las tecnologías digitales, y la que sea dominante determinará cómo serán los usuarios tecnológicos del futuro. Macgilchrist et al. (2019) realizan un ejercicio de "teoría ficción" en ciencias sociales al especular con tres posibles escenarios futuros, en el año 2040, considerando las decisiones políticas, educativas y tecnológicas que nos acercan más a estas configuraciones sociotécnicas. Cada una de las tendencias sobre ciudadanía y juventud guarda relación con estas tres historias. La primera historia, sobre "usuarios suaves y sujetos competentes" sería resultado de fomentar la primera tendencia, la "normativa" con un "enfoque de control". En este escenario, se promueve la adquisición de competencias técnicas y se busca la eficiencia en el uso de herramientas digitales para encajar en el mercado laboral y en una sociedad posdemocrática dominada por corporaciones. La segunda historia se centra en los "nómadas digitales". En ella, la tendencia "participativa" con un "enfoque de libertad" lleva a los usuarios a cultivar el individualismo y la superficialidad mientras explotan las regulaciones estatales y algorítmicas para deslizarse hacia una nueva economía capitalista. La tercera historia, 
sitúa un escenario donde prima la "acción colectiva", en el que las instituciones son espacios para explorar nuevas formas de convivencia más equitativas y sostenibles. Este tercer futuro es el más acorde los objetivos planteados por la investigación, y se propone que la tendencia "cívica" con "enfoque crítico" es la que puede promover de manera más efectiva los procesos de toma de decisiones que acerquen a la juventud a un escenario de esas características.

En lo que respecta al futuro de la educación y su relación con las tecnologías digitales la pandemia de COVID-19 supone un punto de inflexión (Selwyn et al., 2020). Las medidas de confinamiento han obligado a una adaptación forzosa de la enseñanza al ámbito online para poder continuar con su actividad. En esta situación de aceleracionismo tecnológico obligado, la propensión hacia la privatización de la educación digital mediante la utilización de servicios corporativos se está acentuando (Internacional de la Educación, 2020). La pandemia ofrece la cobertura bajo la urgencia coyuntural para que las corporaciones EdTech desplieguen el discurso del solucionismo tecnológico, mientras las instituciones educativas se ven obligadas a tomar decisiones rápidas para paliar la situación de crisis. Esta situación cristaliza en la formación de nuevas redes de poder en el contexto de la pandemia con alianzas entre las corporaciones EdTech y organismos de gobernanza internacional y gobiernos nacionales (Selwyn et al., 2020). Estas cuestiones colocan a la educación social ante la necesidad de desarrollar un programa de alfabetización digital que tenga en consideración los cambios estructurales que la pandemia provoca en el entorno digital.

La revisión de literatura muestra que la concepción dominante de alfabetización mediática, en la que se fundamentan muchas propuestas de alfabetización digital, procede de una definición de hace casi tres décadas. Esta falta de renovación, así como de consideración de las normas predominantes de la cultura digital actual (espectacularización, desconfianza en las instituciones, brecha de intervención), profundizadas por la pandemia, se traduce en una disminución de la relevancia de los proyectos de alfabetización. Esto supone revelar la ideología que subyace a la concepción dominante de la alfabetización (Carmi et al., 2020), y dejar de priorizar la adquisición de habilidades utilitarias con el objetivo de preparar al usuario para su inserción en el mercado laboral. Una alfabetización digital que ponga el foco en la promoción de valores como la intervención, los cuidados, la conciencia crítica, la persistencia y la emancipación puede aumentar el impacto cívico y social de las iniciativas alfabetizadoras. Esta alfabetización digital crítica, además, fomentaría el conocimiento y uso de herramientas y programas digitales de software libre, analizaría las estructuras de poder existentes y promovería el activismo ciudadano autónomo.

Para ello, los programas de alfabetización digital deben hacer más énfasis en competencias relativas a la producción de contenidos y formatos, y a las habilidades críticas para aumentar su impacto cívico y social. También habrán de tener en cuenta tres dimensiones consecutivas. En primer lugar, los componentes estructurales relativos a la influencia de factores socioculturales y socioeconómicos (raza, etnia, lugar de origen, género, lugar de residencia, clase social, etc). En segundo lugar, habilidades técnicas e instrumentales, como prerrequisito para la tercera dimensión: competencias psicológicas, cognitivas y afectivas. Éstas deberían permitir tanto la experimentación con las tecnologías digitales como su contextualización en las relaciones de poder político y económico. 
Al igual que ocurre con la alfabetización digital, en lo que respecta a la educación social la pandemia de COVID-19 puede aseverar los problemas identificados en esta revisión en su relación con las tecnologías digitales: deshumanización y burocratización de sus prácticas profesionales, desconocimiento y falta de competencias, escasez de apoyo institucional y técnico, y exceso de peso de las tecnologías en el trabajo. Las propuestas resultantes de la revisión apuntan hacia una innovación educativa basada en la reapropiación de las tecnologías digitales desde un enfoque convivencial y la profundización en la horizontalidad pedagógica, cuestiones dificultadas por la crisis sociosanitaria. Para evitar el solucionismo tecnológico predominante, el método de aprendizaje basado en la investigación puede otorgar la autonomía necesaria a la educación social para desarrollar sus prácticas profesionales digitales.

De este modo, la educación social puede contribuir a la reducción de la brecha de acceso digital, entendida en términos socioculturales y socioeconómicos, y al desarrollo de los sectores más vulnerables de la población por cuestiones como el género, la clase social, la etnia, etc. Este desarrollo se apoya en procesos de deliberación y toma de decisiones que incluyan a la juventud, la garantización de los derechos digitales, la autoorganización y autogestión de la sociedad civil, y un aumento de la inversión del sector público. Como resultado, la educación social digital puede aumentar su impacto cívico en términos de inclusión, empoderamiento, coaprendizaje y experimentación con la juventud.

\section{Bibliografía}

(Las referencias marcadas con * son las incluidas en la síntesis cualitativa)

-Alonso Puelles, A.; Aparicio, C.; Alonso, D.; González, G.; Prieto, et al. (2017). Uso de herramientas online para incentivar los debates y reflexiones de las y los estudiantes de forma presencial y/o online [Info: eu-repo/semantics/pimcd]. Retrieved from https://eprints.ucm.es/44334/

-Álvaro, A. \& Rubio, R. (2016): "Las TIC en la participación política de los jóvenes", Instituto de la Juventud. [En Línea] Disponible en

https://issuu.com/injuve/docs/tic_participacionpolitica

-Aranda, D.; Sánchez-Navarro, J.; Martínez-Martínez, S. (2014): “El juego digital e Internet como ecosistema lúdico. Jerarquía de medios para el entretenimiento y alfabetizaciones emergentes". Eleá, I. (Ed.) Agentes e Vozes Um Panorama da MídiaEducação no Brasil, Portugal e Espanha. The International Clearinghouse on Children, Youth and Media at Nordicom, p. 219-228.

-Asociación Estatal De Educación Social - ASEDES (2007). Documentos profesionalizadores. ASEDES: Barcelona.

-Aufderheide, P. (1993). "Media Literacy." A Report of the National Leadership Conference on Media Literacy. Aspen Institute. 
-Bard, A.; Söderqvist, J. (2002). Netocracy: The New Power Elite and Life after Capitalism. London: Pearson Education Limited.

-Blum-Ross, A. \& S. Livingstone. (2016): "From youth voice to young entrepreneurs: The individualization of digital media and learning", Journal of Digital and Media Literacy, Vol. 4, pp. 1-2: online.

-Boulianne, S. (2015): "Online news, civic awareness, and engagement in civic and political life", New Media \& Society, Vol. 18, No. 9, pp. 1840-1856.

-boyd, d. (2014). It's Complicated: The Social Lives of Networked Teens. New Haven, CT: Yale University Press.

-Burdge, R. J., \& Vanclay, F. (1996): "Social impact assessment: a contribution to the state of the art series", Impact assessment, Vol. 14, pp. 59-86. DOI:

10.1080/07349165.1996.9725886

-Carmi, E.; Yates, S.; Lockley, E. \& Pawluczuk, A. (2020): "Data citizenship: rethinking data literacy in the age of disinformation, misinformation, and malinformation", Internet Policy Review, Vol. 9, No. 2. DOI: 10.14763/2020.2.1481

-Carretero, S.; Vuorikari, R. and Punie, Y. (2017): "DigComp 2.1: The Digital Competence Framework for Citizens with eight profi- ciency levels and examples of use", EUR 28558 EN. DOI: $10.2760 / 38842$

-Castells, M. 2010. The Information Age: Economy, Society and Culture. Vol. 1: The Rise of the Network Society. Malden, MA: Blackwell Publishers Inc.

-Correa, T. (2015): "Digital skills and social media use: how Internet skills are related to different types of Facebook use among 'digital natives'”, Information, Communication \& Society, Vol. 19, No. 8, pp. 1095-1107.

${ }^{*}{ }^{*}$ Choi, M. (2016): "A concept analysis of digital citizenship for democratic citizenship education in the digital age", Theory and Research in Social Education, Vol. 44, No. 4, pp. 565-607. DOI: 10.1080/00933104.2016.1210549

-Choi, M.; Glassman, M. \& Cristol, D. (2017): "What it means to be a citizen in the internet age: Development of a reliable and valid digital citizenship scale", Computers \& Education, Vol. 107, pp. 100-112. DOI: 10.1016/j.compedu.2017.01.002

-Choi, M.; Cristol, D. \& Gimbert, B. (2018): "Teachers as digital citizens: The influence of individual backgrounds, internet use and psychological characteristics on teachers' levels of digital citizenship", Vol. 121, pp. 143-161. DOI: 10.1016/j.compedu.2018.03.005

-Cohen, C. J. \& J. Kahne (2012). Participatory politics: New media and youth political action. Chicago: MacArthur Foundation.

-*De Lucas, F. \& D’Antonio, S. (2019): "ICT, young people and social work: distances and opportunities", Social Work Education, DOI: 10.1080/02615479.2019.1691163 
-*Diaconu, M., Racovita, L., Carbonero, D. \& Faubert, S. (2019): “Social work educators' perceived barriers to teaching with technology: the impact on preparing students to work with younger clients", Social Work Education, DOI: 10.1080/02615479.2019.1683155

-Fox, J. (2020): "Fake News - the perfect storm: historical perspectives", Historical Research, Vol. 93, No. 259, pp. 172-187. DOI: 10.1093/hisres/htz011

-Freelon, D., Wells, C. \& Bennett, W.L. (2013): "Participation in the Youth Civic Web: Assessing User Activity Levels in Web Sites Presenting Two Civic Styles", Journal of Information Technology \& Politics, Vol.10, No.3, pp. 293-309.

-Fuchs, C. (2014). Social Media: A Critical Introduction. London: Sage

-*Garmendia, M. \& Karrera, I. (2019): "ICT use and digital inclusion among roma/gitano adolescents", Media and Communication, Vol. 7, No. 1, pp. 22-31. DOI:

10.17645/mac.v7i1.1624

-Gillmor, D. (2010). Mediactive. Accesible en http://mediactive.com/book/ [recuperado 23-092018]

-Gilster, P. (1997). Digital literacy. New York, NY: Wiley Computer Pub.

-*Gleason, B. \& Von Gillern, S. (2018): "Digital citizenship with social media: Participatory practices of teaching and learning in secondary education", Educational Technology and Society, Vol. 21, No. 1, pp. 200-212.

-Hargittai, E. (2010): "Digital Na(t)ives? Variation in internet skills and uses among members of the "net Generation'”, Sociological Inquiry, Vol.80, No.1, pp. 92-113.

-Harvey, C. (2016). Using ICT, digital and social media in youth work. National Youth Council of Ireland 2016. Dublin: National Youth Council of Ireland.

-Hibberson, S., Barrett, E. \& Davies, S. (2015). Developing students' digital literacy. Retrieved from https://www.jisc.ac.uk/guides/developing-students-digital-literacy

-*Hitchock, L. \& Young, J. (2016): "Tweet, Tweet!: Using Live Twitter Chats in Social Work Education”, Social Work Education, Vol. 35, No. 4, pp. 457-468. DOI: 10.1080/02615479.2015.1136273

-Hobbs, R. (2010): "Digital and media literacy - A Plan of Action", The Aspen Institute, Washington, D.C. [En Línea] Disponible en

https://www.knightfoundation.org/media/uploads/publication_pdfs/Digital_and_Media_Literacy_A_P lan_of_Action.pdf

-Illich, I. (2011). La convivencialidad. Barcelona: Virus editorial.

-Impero Software \& Digital Citizenship Institute (2016): "White paper. Digital citizenship. A Holistic primer". Retrieved from http://www.digitalcitizenshipinstitute.com/wpcontent/uploads/2016/10/digital-citizenship-a-holistic-primer-v2-1.pdf 
-Internacional de la Educación (2020): "La comercialización y la privatización en y de la educación en el contexto de la COVID-19". Recuperado de: https://ei-ie-al.org/recurso/lacomercializacion-y-la-privatizacion-en-y-de-la-educacion-en-el-contexto-de-la-covid-19

-Ito M. et al. (2009). Hanging out, messing around, geeking out: Living and learning with new media. Cambridge, MA: MIT Press.

-Jenkins, H., R. Purushotma, K. Clinton, M. Weigel, \& A. J. Robinson. (2006). Confronting the challenges of participatory culture: Media education for the 21 st century. Chicago: Mac- Arthur Foundation.

-Jenkins, H. (2016): "Youth Voice, Media and Political Engagement. Introducing the Core Concepts", en $\mathrm{H}$. Jenkins et al, By any means necessary. The New Youth Activism. Nueva York: NYU Press.

-Jones-Jang, S.M., Mortensen, T. \& Liu, J. (2019): “Does Media Literacy Help Identification of Fake News? Information Literacy Helps, but Other Literacies Don't" American Behavioral Scientist. DOI: 10.1177/0002764219869406

-Kaplún, M. (1998). Pedagogía de la comunicación. Madrid: La Torre.

-Kelty, Ch. M. (2008). Two Bits. The Cultural significance of free software. Duke University Press. Accessible en https://twobits.net/pub/Kelty-TwoBits.pdf

-Kleine, D. (2013). Technologies of choice? ICTs, development, and the capabilities approach. Cambridge, MA: MIT Press.

-*Literat, I., Kligler-Vilenchik, N., Brough, M. \& Blum-Ross, A. (2018): "Analyzing youth digital participation: Aims, actors, contexts and intensities", Information Society, Vol. 34, No. 4, pp. 261-273. DOI: 10.1080/01972243.2018.1463333

-*Liubieniene, V. \& Thunqvist, D. (2015): "Media literacy and digital divide: a cross-cultural case study of Sweden and Lithuania", Creativity Studies, Vol. 8, No. 2, pp. 134-148. DOI: $10.3846 / 23450479.2015 .1046407$

-Lovink, G. (2019). Sad by design. London: Pluto Press.

-Macgilchrist, F., Allert, H. \& Bruch, A. (2019): "Students and society in the 2020s. Three future 'histories' of education and technology", Learning, Media and Technology. DOI: 10.1080/17439884.2019.1656235

-*Martens, H. \& Hobbs, R. (2015): "How Media Literacy Supports Civic Engagement in a Digital Age", Atlantic Journal of Communication, Vol. 23, No. 2, pp. 120-127. DOI: 10.1080/15456870.2014.961636

-Middaugh, E. \& Kahne, J. (2013): "New media as a tool for civic learning", Comunicar, Vol. 20, No. 40, pp. 99-107. 
-*Mihailidis, P. (2018): "Civic media literacies: re-Imagining engagement for civic intentionality”, Learning and Media Technology, Vol. 43, No. 2, pp. 152-164. DOI: 10.1080/17439884.2018.1428623

-Mihailidis, P. \& Thevenin, B. (2013): "Media Literacy as a Core Competency for Engaged Citizenship in Participatory Democracy", American Behavioral Scientist, Vol. 57, No. 11, pp.1611-1622.

-Mihailidis, P. \& Viotty, S. (2017): "Spreadable Spectacle in Digital Culture: Civic Expression, Fake News, and the Role of Media Literacies in 'Post-Fact' Society", American Behavioral Scientist, Vol. 61, No. 4, pp. 451-454. DOI:

10.1177/0002764217701217

-Moher D, Liberati A, Tetzlaff J, Altman DG, The PRISMA Group (2009). "Preferred Reporting Items for Systematic Reviews and Meta-Analyses: The PRISMA Statement", PLoS Med, Vol. 6, No. 7. DOI: 10.1371/journal.pmed1000097

-Morales, R. et al. (2016): "The New Frontier of Digital Inequality. The Participatory Divide", Revista Española de Investigación Sociológica, No. 156, pp. 97-116.

-Morozov, E. (2011). The Net Delusion: the Dark Side of Internet Freedom. New York, USA: Public Affairs.

-Morozov, E. (2013). To save everything, click here: the folly of technological solutionism. New York, USA: Public Affairs.

-Mossberger, K., Tolbert, C. J. \& McNeal, R. S. (2008). Digital citizenship: The internet, society, and participation. Cambridge, MA: MIT Press.

-Okoli, C., Schabram, K. (2010): "A Guide to Conducting a Systematic Literature Review of Information Systems Research" . Disponible en

https://papers.ssrn.com/sol3/papers.cfm?abstract_id=1954824

-*Panke, S. \& Stephens, J. (2018): "Beyond the echo chamber: Pedagogical tools for civic engagement discourse and reflection", Educational Technology and Society, Vol. 21, No. 1 , pp. 248-263.

-*Pawluczuk, A., Webster, G., Smith, C. \& Hall, H. (2019): "The Social Impact of Digital Youth Work: What Are We Looking For?", Media and Communication, Vol. 7, No. 2, pp. 59-68. DOI: 10.17645/mac.v7i2.1907

-*Pereira, S., Fillol, J. \& Moura, P. (2017): "Young people learning from digital media outside of school: The informal meets the formal”, Comunicar, Vol. 27, No. 58, pp. 41-50. DOI: 10.3916/C58-2019-04

-*Pérovic, J. (2015): "Media literacy in Montenegro", Media and Communication, Vol. 3, No. 4, pp. 91-105. DOI: 10.17645/mac.v3i4.335 
-*Pötzsch, H. (2019): "Critical digital literacy: Technology in education beyond issues of user competence and labour-market qualifications", TripleC, Vol. 17, No. 2, pp. 221-240. DOI: 10.31269/triplec.v17i2.1093

-Ranieri, M., Rosa, A. \& Manca, S. (2016): "Unlocking the Potential of Social Media for Participation, Content Creation and e-Engagement. Students' Perspectives and Empowerment", in E.L. Brown, A. Krasteva, \& M. Ranieri (EDs), E-learning and Social Media: Education and Citizenship for the Digital 21st Century, pp. 223-248, Charlotte, NC: Information Age Publishing. ISBN 9781681234281.

-Sampedro, V. (2005). 13-M: Multitudes online. Madrid: Catarata.

-Sampedro, V. (2018). Dietética Digital para adelgazar al Gran Hermano. Barcelona: Icaria. Disponible en https://dieteticadigital.net/dietetica-digital-libre/

-Sánchez-Navarro, J. \& Aranda, D. (2012): "Messenger and social network sites as tools for sociability, leisure and informal learning for Spanish young people", European Journal of Communication, Vol. 28, No. 1, pp. 67-75.

-Selwyn, N., Macgilchrist, F. \& Williamson, B. (2020): “Techlash \#1. Digital education after COVID". Recuperado de: http://der.monash.edu.au/lnm/techlash-1-is-out-digital-education-aftercovid-19/

-Siddaway, A., Wood, A., \& Hedges, L. (2019): "How to Do a Systematic Review: A Best Practice Guide for Conducting and Reporting Narrative Reviews, Meta-Analyses, and Meta-Syntheses", Annual Review of Psychology, Vol. 70, No. 9, pp. 1-24.

-Simanowski, R. (2018). Stumme Medien: Vom Verschwinden der Computer in Bildung und Gesellschaft. Berlin: MSB Matthes \& Seitz.

-Srnicek, N. (2016). Platform Capitalism. Cambridge: Polity Press.

-Stoilova, M.; Livingstone, S. \& Kardefelt-Winther, D. (2016): "Global Kids online:

Researching children's rights globally in the digital age", Global Studies of Childhood, Vol. 6, No. 4, pp. 455-466. DOI: 10.1177/2043610616676035

-*Tugtekin, E. \& Koc, M. (2019): "Understanding the relationship between new media literacy, communication skills, and democratic tendency: Model development and testing", New Media \& Society, DOI: 10.1177/1461444819887705

-Van Deursen A. \& Van Dijk J. (2011): "Internet skills and the digital divide", New Media \& Society, Vol. 13, No. 6, pp. 893-911. DOI: 10.1177/1461444810386774

-Vuorikari, R., Punie, Y., Carretero Gomez S., Van den Brande, G. (2016). DigComp 2.0: The Digital Competence Framework for Citizens. Update Phase 1: The Conceptual Reference Model. Luxembourg Publication Office of the European Union. EUR 27948 EN. Doi:10.2791/11517

-*Young, J. (2015): "Assessing New Media Literacies in Social Work Education: The Development and Validation of a Comprehensive Assessment Instrument", Journal of 
Technology in Human Services, Vol. 33, No. 1, pp. 72-86. DOI: $10.1080 / 15228835.2014 .998577$

-*Yue, A., Nekmat, E. \& Beta, A. (2019): "Digital Literacy Through Digital Citizenship: Online Civic Participation and Public Opinion Evaluation of Youth Minorities in Southeast Asia", Media and Communication, Vol. 7, No. 2, pp. 100-114. DOI: 10.17645/mac.v7i2.1899

-`Zorn, I. \& Seelmeyer, U. (2017): “Inquiry-Based Learning about Technologies in Social Work Education", Journal of Technology in Human Services, Vol. 35, No. 1, pp. 49-62.

DOI: 10.1080/15228835.2017.1277913 